myeloperoxidase enzyme. This affinity could explain why myeloperoxidasederived products modify an extraordinary amount of apoA-I compared to other proteins. These modifications of apoA-I interfere with the ability of the molecule to bind and remove lipids, thus leading to increased lipid accumulation in the arteries of patients with cardiovascular disease.

Because the source of the modifying agents (myeloperoxidase products) is the macrophages in the atherosclerotic lesions, this constitutes a positive feedback loop to intensify the inflammation. The feedback loop probably evolved to fight infection and is now deleterious because there is no infecting agent. In cardiovascular disease, the inflammatory reaction is initiated by low density lipoprotein (LDL)-derived oxidized lipids that initiate the same cascade of cellular events as if there were an infectious agent present.

In animals, HDL is often the major source of plasma cholesterol. But in adult humans the major source of plasma cholesterol is not the apoA-I-containing proteins (HDL), but rather the apolipoprotein $\mathrm{B}$ (apoB)-containing lipoproteins (LDL). Throughout the particle's life in plasma,
apoB remains with the lipoprotein particle to which it was originally attached. ApoB also has a heparin-binding site that causes apoB-containing proteins to avidly bind to the matrix in the space just beneath the single endothelial cell layer that lines the lumen of arteries. As a result, the concentration of apoB in even normal arteries is twice that in plasma.

Unlike apoB, apoA-I does not stay with one particle, but moves from one particle to another. Normal apoA-I also does not have a heparin-binding site and there is normally more apoA-I in the plasma than in the arteries. But in atherosclerotic arteries, apoA-I accumulates in abundance ${ }^{11}$. Perhaps the accumulation of apoA-I in atherosclerotic arteries is related to myeloperoxidase-induced changes in the protein, which could make apoA-I able to tightly bind the artery wall.

Pharmaceutical companies are currently testing agents to raise HDL cholesterol levels in humans by blocking normal HDL metabolic pathways ${ }^{12}$. The work cited here suggests that this strategy, although it may raise HDL cholesterol levels, may not improve clinical outcomes as a single therapy. Statin therapy modestly reduces myeloperoxidase products in plasma ${ }^{9}$ and modestly improves the inflammatory properties of $\mathrm{HDL}^{3}$, but not to the levels seen in normal humans. The combination of statin therapy and therapy to raise HDL levels may prove useful if the net effect is to produce anti-inflammatory HDL. If the combination fails to render the increased HDL sufficiently anti-inflammatory, the combination may not significantly improve clinical outcomes.

1. Navab, M. et al. J. Lipid Res. 45, 993-1007 (2004).

2. Navab, M. et al. J. Lipid Res. 42, 1308-1317 (2001).

3. Ansell, B.J. et al. Circulation 108, 2751-2756 (2003).

4. Zheng, L. et al. J. Clin. Invest. 114, '-yyy (2004).

5. Navab, M. et al. J. Lipid Res. 41, 1481-94 (2000).

6. Navab, M.et al. J. Lipid Res. 41, 1495-1508 (2000)

7. Navab, M. et al. Circulation 105, 290-292 (2002).

8. Navab, M. et al. Circulation 109, r120-r125 (2004).

9. Shishehbor, M.H. et al. JAMA 289, 1675-1680 (2003).

10. Brennan, M.L.et al. N. Engl. J. Med. 349, 1595-1604 (2003).

11. Mackness, B., Hunt, R., Durrington, P.N. \& Mackness M.I. Arterioscler. Thromb. Vasc. Biol. 17, 1233-1238 (1997).

12. Brousseau, M.E. et al. N. Engl. J. Med. 350, 1505-1515 (2004).

\title{
Mutated matrix gene mars macula
}

Macular degeneration results from wear and tear over the years in the center of the retina, and afflicts mainly the elderly. Genetics is suspected to be a factor in this degenerative disease; for instance, mutations in the gene encoding fibulin-3 contribute to an earlyonset form. In the 22 July issue of New England Journal of Medicine, Edwin Stone et al. implicate mutations in a related gene, encoding fibulin-5, in late-onset disease.

The researchers sequenced six genes in the fibulin family and found missense mutations in the fibulin- 5 gene in 7 of 402 patients with age-related macular degeneration. The mutations were not the same in each individual, but six mutations deviated from conserved sequences in several species. Many variations in the other fibulin genes were also found in the patients, although larger studies will be required to pin down a concrete association with disease.

Fibulin-5, an extracellular matrix protein,

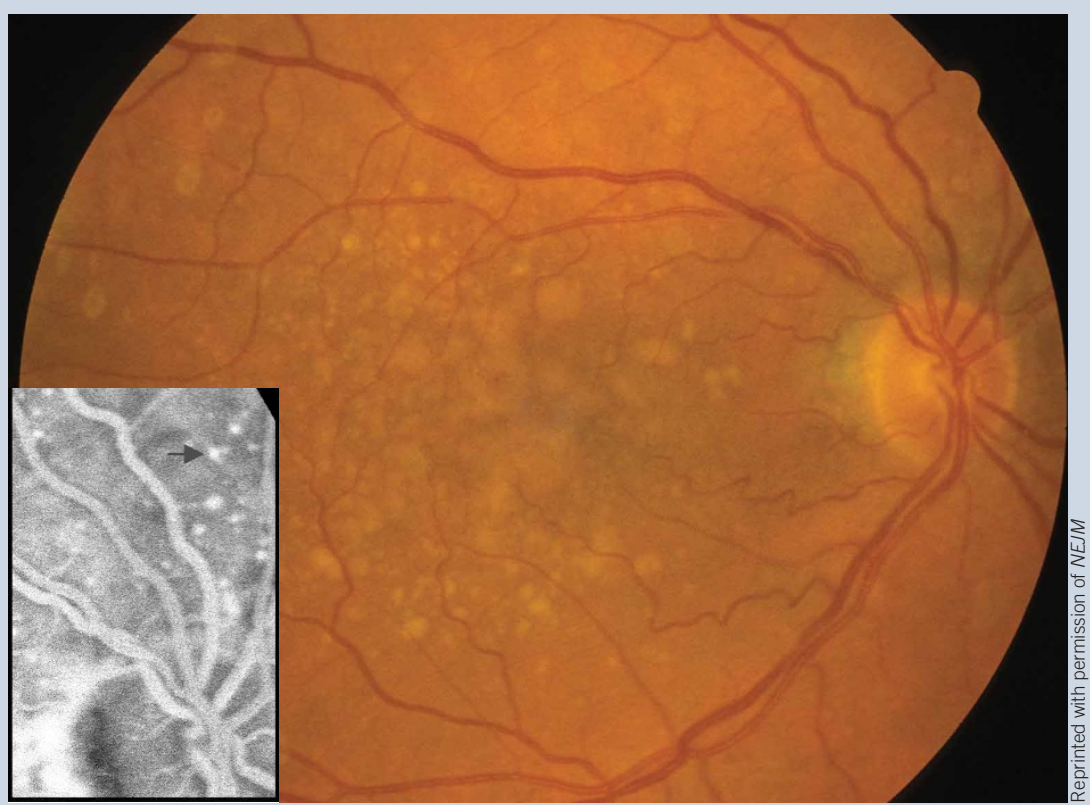

has binding sites for numerous basement membrane proteins and is required for polymerization of elastin. Elastin is a major component of a structure in the eye that gives rise to drusen, small bits of protein and lipid associated with this disease (Shown is an eye of a patient with a mutation in the gene for fibulin-5. Macula occupies central half of figure, optic nerve head is on right. Inset, arrow points to prominent drusen visible with fluorescein angiography.) 